\title{
COMPRÉHENSION DES MÉDIAS ET SAVOIRS
}

\author{
Pierre Molinier ${ }^{1}$
}

Par nature, les savoirs construits au contact des médias se classent dans le domaine de l'informel, ou plus exactement, du non formel. Ils ne relèvent pas d'une organisation validée et hiérarchisée en terme de programmes. Ils ne font pas l'objet d'une évaluation et donc d'une valorisation dans un cadre institutionnel. Pour autant, le quotidien de chaque individu est fortement tributaire de ces constructions issues des contenus médiatiques dans des domaines aussi variés que la météorologie, la technologie et les techniques, les sciences biologiques (ESB, embryologie), l'histoire (guerre d'Algérie, conflit israélo-palestinien), l'économie, le politique...

Un examen des définitions, notamment celle de J. Beillerot, atteste que ces constructions peuvent bien relever des savoirs : "ce qui, pour un sujet, est acquis, construit et élaboré par l'étude ou l'expérience. Résultat d'une activité d'apprentissage quelles que soient la nature et la forme de celui-ci (...) le savoir s'actualise dans des situations et dans des pratiques" 2 . On constate cependant que ces savoirs s'actualisent peu dans le champ des formations formelles, plus particulièrement scolaires. Cette intégration ne se produit-elle pas en

\footnotetext{
1 Maître de conférences à l'ESAV, LARA, Université de Toulouse 2.

2 J. BeILlerot, "Savoir" in Dictionnaire encyclopédique de l'éducation et de la formation, Paris, Nathan Université, pp. 890-891.
} 
raison du manque de structuration théorique ou de la faible valeur heuristique qui leur sont attribués?

Lors d'études de réceptions télévisuelles auprès d'enfants de 8/11ans, il nous est apparu cependant que ces démarches ne produisaient en fait que peu de savoirs effectifs à cet âge là. L'investissement proprioceptif, même fort, ne pouvait compenser le déficit d'appropriation cognitive, fréquemment observé. En reprenant les trois états d'un objet cognitif distingués par M. Linard : "son état d'information (donnée brute objective), son état de connaissance (information subjectivement assimilée par un individu) et son état de savoir (connaissance socialement validée et objectivée dans un document ou un discours)"', il semble que les enfants aient des difficultés à franchir le cap du dernier de ces états. Nous faisons l'hypothèse que la carence partielle de compréhension, obère la construction d'apprentissages, qui s'ils étaient pleinement intégrés et opérationnels pourraient rejoindre partiellement les pratiques scolaires. D'autant que ces pratiques sont de plus en plus ouvertes et tolérantes à ces acquis extérieurs.

C'est dans ce but que nous avons observé les démarches de compréhension des enfants face aux médias pour en repérer les diverses fonctionnalités afin d'intervenir de façon éducative dans leur appropriation. En retour, nous espérons une efficience et une diversification de savoirs construits afin que la frontière entre le non formel et l'informel soit de plus en plus poreuse, que les expériences de téléspectateur et d'élève puissent s'étayer réciproquement.

Cette perspective de donner à l'enfant des ressources pour mieux comprendre, rejoint pleinement les priorités éducatives établies par $\mathrm{E}$. Morin : "le problème de la compréhension est devenu crucial pour les humains. Et, à ce titre, il se doit d'être une des finalités de l'éducation (...) L'on retrouve ici la mission proprement spirituelle de l'éducation : enseigner la compréhension entre les humains est la condition et le garant de la solidarité intellectuelle et morale de l'humanité"'.

1 M. LINARD, "Les technologies de l'information et de la communication en éducation : un pont possible entre faire et dire" in L'état de l'enfance en France, les jeunes et les médias, Paris, Hachette, 2000, pp. 151-176.

2 E. MORIN, Les sept savoirs nécessaires à l'éducation du futur, Paris, Éd. du Seuil, 2000 , p. 103. 


\section{Le cadre de la recherche-action}

Depuis six années, nous avons engagé une recherche-action "l'Enfant et les écrans"' sur les rapports entre le monde de l'école et celui des médias. Sa mise en œuvre se déroule dans 40 classes maternelles et élémentaires du département du Tarn et vise une réelle éducation aux médias. Le dispositif méthodologique retenu, nous amène à poursuivre conjointement deux objectifs comme le précise M.-R. Verspieren "la recherche-action de type stratégique se présente comme une méthode de recherche qui attache autant d'importance à l'action comme moyen de transformation de la réalité, qu'à la recherche comme moyen de connaître cette réalité et de développer cette connaissance"2.

L'action consiste à intégrer dans le quotidien scolaire les ressources matérielles, langagières, cognitives et formatives nécessaires, afin que les médias constituent de réels objets et supports d'enseignement. Cette intégration veille par ailleurs à créer les conditions de transfert de certains acquis vers les pratiques sociales de l'enfant. Ces formations visent à construire une continuité entre les dispositifs des principaux médias diffusés sur écran : télévision, cinéma, multimédia, tout en repérant par comparaison, leurs singularités sémio-pragmatiques respectives. Les démarches pédagogiques engagées par les enseignants, sont régulièrement échangées, critiquées, évaluées, afin de constituer par mutualisation des ressources praxéologiques communes et diffusables.

Ces pratiques constituent un potentiel de situations d'observation et d'expérimentation qui alimentent la recherche théorique centrée essentiellement dans un premier temps, sur les enjeux langagiers (audiovisuel, oral, écrit) dans la construction de sens des divers messages étudiés. En contrepartie, les connaissances acquises ou en voie de validation alimentent le champ de l'action dans les classes.

1 Cette action est conduite dans le cadre du Laboratoire d'Études Audiovisuelles (LAVEA) en partenariat avec l'École Supérieure d'Audiovisuel de l'Université Toulouse II (ESAV), l'IUFM de Midi-Pyrénées, l'association MédiaTarn et l'Inspection Académique du Tarn.

2 M.R. VERSPIEREN, Recherche-action de type stratégique et science(s) de l'éducation, Paris, L'Harmattan, 1990, p. 86. 


\section{La méthodologie de la recherche-action}

Lors des observations et des enregistrements menés auprès d'enfants de divers âges confrontés à des messages télévisuels en milieu scolaire, il est apparu nettement que la construction de savoirs était fréquemment handicapée par un déficit de compréhension tant sur le plan sémiotique, pragmatique, que sémantique ${ }^{1}$. Le terme de compréhension étant entendu en nous référant aux travaux d'U. $E \mathrm{Co}^{2}$ comme l'actualisation sémantique des divers paramètres du message par le récepteur et son activité de coopération. Activité qui s'organise à partir de modèles inférentiels résultant de processus d'interaction entre les trois grandes composantes que sont: le message audiovisuel, le contexte de réception et le sujet récepteur. Les significations construites par ce dernier sont intégrées et assimilées à ses connaissances antérieures.

L'observation empirique des pratiques des élèves nous a permis de poser comme hypothèse :

a. l'acte de compréhension est un préalable et/ou un corollaire à toute construction de savoirs formalisables ;

b. la maîtrise des langages audiovisuel, oral et écrit joue un rôle fondamental dans l'acte de compréhension et dans la formalisation des savoirs ${ }^{3}$;

c. l'acte de compréhension rendu opaque par la fulgurance de son effectuation peut partiellement s'observer au travers des formes langagières qui l'expriment.

Nous rendrons compte ici seulement de cette dernière hypothèse en présentant le modèle bâti à partir de l'analyse des séquences de sémiogenèse collective enregistrées dans les classes. Ces séquences sont systématiquement conduites dans le réseau, après une première

1 Le terme de déficit provient du fait que dans le contexte scolaire le degré d'exigence et d'explicitation des perceptions et des constructions cognitives est plus grand. Ce qui dans le champ de la réception privée relève de l'intuitif et de l'implicite, se révèle à l'épreuve de la formalisation comme déficient ou trop fragile.

2 U. ECO, Les limites de l'interprétation, Paris, Grasset, p. 36.

3 Cette hypothèse s'origine dans l'affirmation de R. Barthes "lire c'est trouver des sens, et trouver des sens c'est les nommer" (S/Z, Paris, Éd. du Seuil, coll. "Point", p. 17) et se voit confirmée par les travaux de P. Charaudeau "le sens n'est pas donné par avance il résulte d'une construction langagière" (Le discours d'information médiatique, Paris, INA Nathan, 1997, p. 40). 
réception d'un nouveau message audiovisuel. Le groupe classe, transformé en réelle communauté interprétative, partage les perceptions et les productions de sens individuelles en tentant de reconstruire la cohérence interne du message dans la continuité de son déroulement. On notera que cette situation pédagogique efficiente, née des impératifs de l'action constitue aussi une situation de production de savoirs particulière, confirmant les propos de J.P. Resweber "la recherche-action est inséparablement une action de recherche et une recherche d'action"'.

Notre démarche inductive, ancrée sur l'observation des productions de sens des élèves s'est articulée sur des modèles abstraits que nous avons transformés ${ }^{2}$. Après une nécessaire réorganisation de ceux-ci, les corroborations ou réfutations apportées par les éléments empiriques ont permis une opérationnalisation. C'est donc de cette double dynamique de l'action et de la théorisation qu'est issu le modèle que nous proposons. Malgré l'attention portée à la méthode nous ne perdrons pas de vue cependant la mise en garde de G. Bramaud du Boucheron qui conserve toute son actualité "il ne peut y avoir de modèles de compréhension exact vu l'état des connaissances en neurophysiologie et la diversité des cognitions humaines"3.

Nous attribuerons donc au modèle présenté ci-dessous, la valeur d'une représentation intermédiaire susceptible d'orienter l'action pédagogique, notamment en structurant les situations qui permettent à l'enfant d'apprendre à mieux comprendre. Les progrès enregistrés dans les réalisations cognitives des élèves, semblent valider au moins partiellement ses possibilités de transformation du réel.

\section{Les modalités de la compréhension}

Le modèle que nous proposons ne considère qu'une part de la démarche globale de compréhension, qui consiste donc en une

1 J.P. RESWEBER, La recherche-action, Paris, PUF, Que sais-je ? 1995, p. 11.

2 U. Eco, Lector in fabula, Paris, Grasset, 1985, p. 88 et J. Giasson, La compréhension en lecture, Bruxelles, De Boeck Université, 1990, p. 16. Ces modèles se sont avérés très ouverts, prévus pour la compréhension des textes écrits ; ils ont permis par des transformations profondes d'intégrer les particularités des messages audiovisuels.

3 G. Bramaud du Boucheron, La mémoire sémantique de l'enfant, Paris, PUF, 1981, p. 244. 
actualisation par le sujet récepteur d'une partie plus ou moins importante du message dans le cadre d'une action entreprise à la finalité plus ou moins explicite. Pour engager cet acte complexe et soudain, le sujet récepteur va utiliser les structures qu'il a construites indépendamment du message auquel il est exposé ou qu'il organise à l'occasion de cette rencontre singulière. Deux grands types de structures sont habituellement répertoriées, affectives et cognitives. Les particularités de la réception audiovisuelle nous incitent à rajouter un troisième, relevant du sensori-moteur en raison de la sollicitation du corps du spectateur. P. Valéry notait déjà l'impact du "neuro-viscéral" sur l'acte de lecture et divers auteurs ont confirmé les répercussions du proprioceptif sur la réception cinématographique ou télévisuelle (S. Calbo, S. Tisseron, V. Le Goaziou).

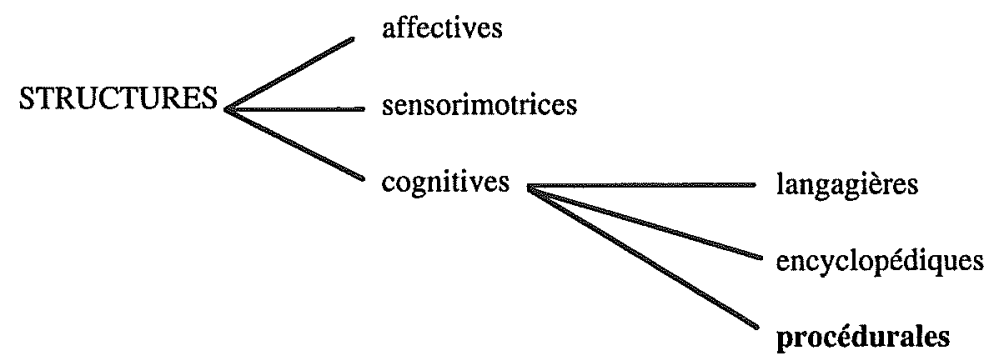

Aujourd'hui les investissements du groupe s'orientent vers une intégration plus marquée des structures sensorimotrices dans les expérimentations pédagogiques, cependant nous ne sommes pas encore suffisamment avancés pour en rendre compte ici. Le modèle est échafaudé dans le domaine du cognitif, en repérant trois grands types de connaissances qui interfèrent sur les productions de sens du sujet.

\section{a. Les connaissances langagières}

La plus ou moins grande maîtrise des langages audiovisuels produit des différences nettes dans la distanciation aux représentations visuelles ou sonores. La comparaison entre enfants aux niveaux d'expertises différents, témoigne de l'enjeu de cette compétence. 
Pour ce qui est de la maîtrise de l'oral, nous avons signalé son importance dans la compréhension '. D'autres travaux confirment ces conclusions tant dans la formulation individuelle que dans les interlocutions du groupe classe (Masselot-Girard, Grandaty, GarciaDebanc).

Les possibilités de se référer au monde de l'écrit permettent d'affiner la classification des produits par genre, et d'ajuster son comportement à la "promesse" de ces genres tout en mesurant la complémentarité de ces deux supports d'expression.

Dans ces trois domaines langagiers, la maîtrise s'exprime tout autant sur le plan sémiotique, pragmatique que sémantique. Les pratiques de classe à partir de l'étude des messages audiovisuels s'avèrent particulièrement riches pour les observations et constructions métalangagières.

\section{b. Les connaissances encyclopédiques}

Ce concept développé par U. Eco met en avant le tissage de connaissances interconnectées, acquis dans l'accumulation des expériences et des découvertes de tout individu. Parmi celles-ci, on retiendra les divers savoirs construits à partir du monde qu'il soit réel ou fictionnel, en repérant la part de plus en plus importante que prennent les médiations audiovisuelles dans la constitution de ces savoirs. Nombre de ceux-ci sont reliés par des scénarios (U. Eco) ou schémas (J. Giasson, Rumelhart) qui structurent les diverses données en types. Ainsi faire des courses dans un supermarché ou participer à l'anniversaire d'un ami supposent un ensemble d'actions et d'éléments factuels prévisibles. Cette organisation peut-être sollicitée uniquement à partir d'un seul facteur représentatif, le caddie pour les courses en supermarché par exemple. Les représentations audiovisuelles renvoient à ces scénarios du réel mais aussi à ceux provenant des récits fictionnels, ils en créent par moment de spécifiques (émissions de type "big brothers").

La rencontre approfondie de divers messages organise, mois après mois, des savoirs d'une autre nature, relatifs aux processus de la création et des engagements esthétiques télévisuels, cinématographiques, multimédias. Les produits étudiés servent de balises pour intégrer par référence, les nouveaux. Progressivement, par des renvois

1 P. Molinier, "La formation du spectateur ou le suicide consenti du pédagogue", Entrelacs, $\mathrm{n}^{\circ}$ 4, Université Toulouse 2, janvier 2002, pp. 145-161. 
fréquents, se structure une culture audiovisuelle débordant largement celle acquise au seul contact des émissions les plus fréquentées socialement. L'extension de cette encyclopédie s'hybride naturellement avec celles provenant des disciplines scolaires, leur donnant souvent une assise plus concrète.

\section{c. Les connaissances procédurales}

Ces connaissances relèvent davantage de savoir-faire que les précédentes et peuvent se décliner en terme de processus comme des suites d'opérations aboutissant à des résultats divers. Notre expérimentation nous a permis d'isoler six catégories de processus que nous décrirons ici plus précisément. Pour en rendre la présentation moins austère, nous nous appuierons sur un film d'animation l'aigle de la série Bestiaire ${ }^{1}$ exploitée dans le réseau. Cet épisode d'une durée de $2 \mathrm{mn} 30^{\prime}$ présente quelques caractéristiques du rapace, mêlant les données scientifiques à une narration non dénuée d'humour.

Ces processus ne peuvent en aucun cas être présentés dans une chronologie ou séquentialité impossibles à établir mais davantage dans une concomitance partielle et labile, liée à la singularité de chaque acte de compréhension.

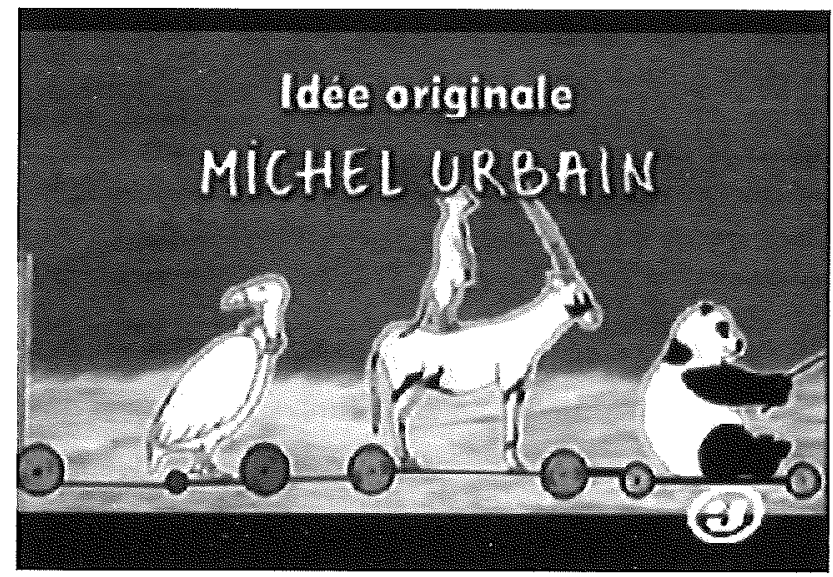

1 Série réalisée par M. Urbain en 1997, coproduite par la RTBF et depuis diffusée fréquemment sur Canal $\mathrm{J}$. 

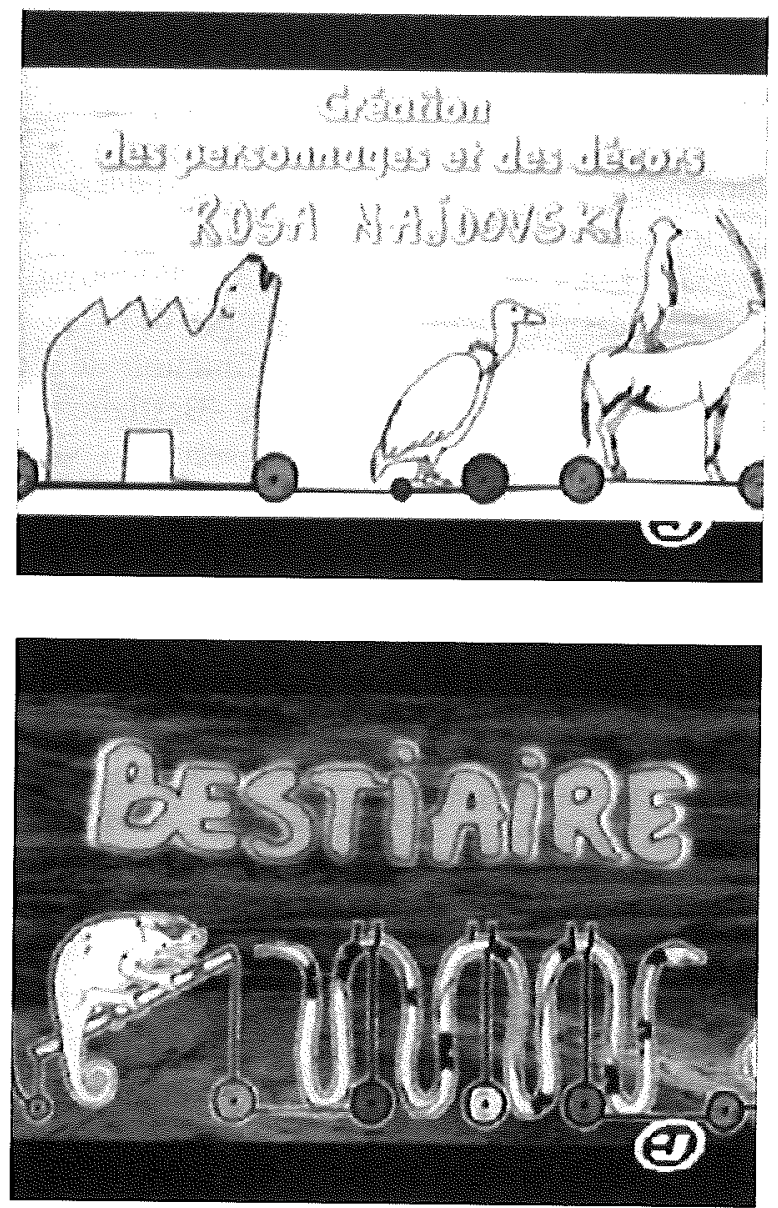

\section{Le modèle des connaissances procédurales}

\section{a. Les processus perceptifs}

Pour saisir leur fonctionnement, il est nécessaire de revenir sur la nature particulière de l'expression audiovisuelle dont nous avons démontré dans de précédents travaux son aspect composite. Constitués de cinq matériaux de base distincts : l'image, les traces graphiques, la voix, les bruits, la musique, ces messages sont perçus par amalgame de deux ou trois d'entre eux. L'œil et l'oreille à partir de ces stimuli transmettent des informations afférentes vers le cortex mais en préalable ou en retour reçoivent des aires spécialisées et multisensorielles des informations efférentes. Il en résulte, comme l'a 
démontré le Groupe $\mu$, que la perception est sémiotisante parce que reliée aux autres processus de compréhension.

Elle est orientée vers l'extraction de figures signifiantes par la segmentation d'unités, qui peuvent différer fortement d'un spectateur à l'autre, confrontés à un même fragment complexe comme le générique de la série. Sous la forme d'un film d'animation dessiné, il comporte une succession d'animaux portés par des wagons, défilant sur un fond d'écosystème changeant en fonction de l'origine des animaux, steppe, prairie, banquise... Le titre "Bestiaire" et les crédits artistiques sont accompagnés d'une chanson à la voix ironique, commentant l'action représentée. Certains enfants ne repèrent que les animaux dont rend compte la chanson, d'autres construisent l'association entre certains animaux et leur milieu, d'autres encore lisent les informations écrites en les reliant à quelques éléments iconiques. Aucun n'est capable de rendre compte de la totalité des unités signifiantes, ou de donner une cohérence globale autre que fonctionnelle au générique. La perception apparaît ici comme fragmentaire et multisensorielle.

En retour l'habileté sémiotique influence ou oriente la sélectivité de la saisie perceptive. La chauve-souris présentée tête en bas sur le wagon final ne sera vue et reconnue par la plupart des élèves, qu'après que le contexte de nuit environnante eut été repéré. Ainsi c'est un syntagme nuit/animal nocturne qui est institué, rappelant l'importance de la préorganisation des données issues de l'acte perceptif. Acte complexe qui synthétise les résultantes des capteurs sensoriels, des aires corticales primaires et secondaires, des données mémorisées, de la collocation d'attention du sujet et de la finalité de la tâche dans lequel il est engagé, par une extraction de figures orientée vers la reconnaissance d'objet et par la segmentation limitée d'unités signifiantes réunissant plusieurs objets.

\section{b. Les microprocessus}

Ils ont pour fonction l'organisation des percepts en unité supérieures, des syntagmes pertinents combinant plusieurs objets segmentés précédemment ou plusieurs segments. Par exemple, les divers animaux identifiés isolément seront associés aux wagonnets et à leur déplacement, constituant l'idée du train en l'absence cependant de toute motrice. Par confrontation aux types (Groupe $\mu$ ) ou aux schèmes (Piaget) les microprocessus favorisent l'actualisation sémantique des associations construites et orientent la saisie du sensible vers le 
concept. R. Lawler privilégiant l'iconique propose le concept de micro-vues. A partir de l'aspect foncièrement disparate des différents éléments sensori-moteurs et perceptifs, les automatismes neurophysiologiques constituent des entités hétérogènes: "du véritable «pandémonium» de micro-vues interactives, conflictuelles qui se réorganisent constamment par effet réciproque, ce n'est que progressivement qu'émergent, par déconnections et reconnections liés à des sauts brutaux «d'intuition», les macro-procédures d'un plus haut niveau d'intégration cognitive"'. L'insistance sur la très grande labilité fonctionnelle caractérise la constitution des micro-vues, rendues composites du fait même de la matière audiovisuelle.

L'autre particularité de microprocessus relève de l'intuition qui régit les diverses associations. Certaine opérations cognitives intuitives s'originent à ce niveau comme la comparaison (de la taille des animaux), certaines projections métaphoriques (le roi-lion avec sa couronne) ou métonymiques (l'ombre de l'aigle) ou encore, comme le précise J.-P. Meunier ${ }^{2}$, les différentes modalités d'ajustement focal (plongée et contre-plongée absolues sur la famille aigle). C'est donc une forme de compréhension intuitive qui s'origine dans cette préorganisation mouvante.

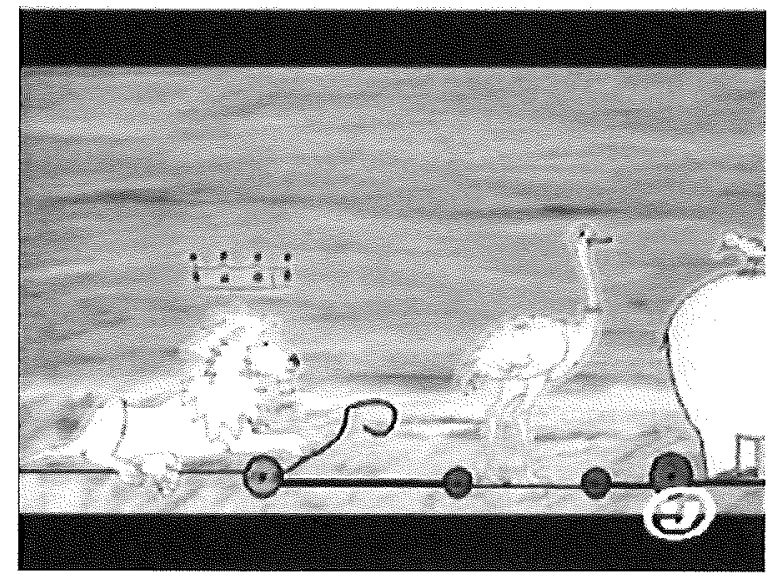

1 R. LAWLER, Computer Experience and cognitive Development, a chil's learning in a computer culture, Chichester, Ellis Horwood, 1985, p. 154.

2 J.-P. MEunier, "Connaître par l'image", Recherches en communication, $\mathrm{n}^{\circ} 10$ (Image(s) et cognition), 1998, pp . 35-75. 

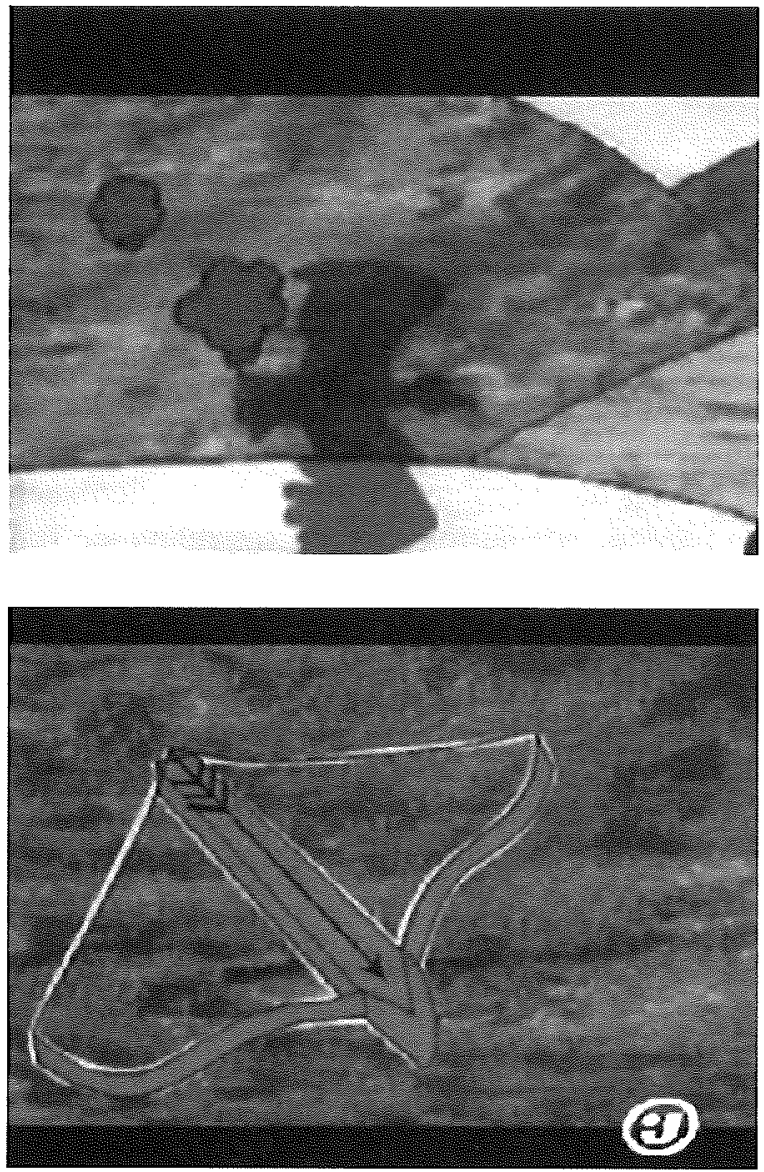

\section{c. Les processus d'intégration}

Ils interviennent essentiellement dans un environnement local, le plan, l'écran ou la microséquence, en créant des relations entre les éléments segmentés et actualisés précédemment. Les concepts formés sont interreliés en réseau d'ordre plus ou moins abstraits (schémas) ${ }^{1}$ par des opérations logico-langagières de nature inférentielle (déduction, induction). Les comparaisons, métaphores et métonymies engagées à ce stade procèdent de ces activités cognitives. Elles diffèrent des précédentes par le recours moindre à l'intuition (afin de

1 "Les schémas, en quelque sorte représentent les stéréotypes de ces concepts. Pour simplifier ils sont comme des modèles du monde extérieur" (D. RUMELHART et D. NORMAN, La psychologie, Paris, Larousse, coll. "Textes essentiels", 1995, p. 310 . 
signaler l'efficacité meurtrière du chasseur, la transformation de l'aigle en arbalète tirant sur la proie construit métaphoriquement une propriété de l'animal en l'isolant). On retrouve ici le fonctionnement de la "connaissance par icône" finement décrite par J.-P. Meunier "comment de nouvelles comparaisons d'images à images induisent de nouvelles assimilations et différenciations conduisant à la formation d'unités plus importantes (...) englobant les précédentes et les enchâssant dans des hiérarchies d'unités signifiantes"l. Ces connections multiples se tissent en prenant appui sur un ensemble de sélections contextuelles, circonstancielles ou encyclopédiques, pour une compréhension du fragment. Ainsi la représentation de la courbe de fréquences du cri de l'aigle sur fond de prairie a fait problème pour des enfants de cet âge là. Les échanges entre eux, pour désambiguïser cette construction permettent d'observer quelques stratégies :

- repérage de la synchronie entre les modulations du cri et de la courbe (mise en confrontation d'éléments pertinents relevant de l'audio-vision) ;

- reconnaissance d'un schéma en raison du caractère scientifique du film et de son insertion dans le cadre de la classe (utilisation du contexte, des circonstances énonciatives et de la "promesse" du genre - F. Jost) ;

- comparaison avec les fréquencemètres de leur chaîne Hi-Fi (recours à l'encyclopédie personnelle).

En assimilant l'image problème au connu, les enfants sélectionnent et identifient des topics ${ }^{2}$ dans la totalité composite du message, constituant ainsi des ensembles homogènes d'éléments sémiopragmatiques vectorisés : les isotopies.

\section{d. Les macroprocessus}

Ils structurent la globalité du message en grandes unités qui permettent un repérage topologique, mais aussi sémantique et fonctionnel. Leur découpage résulte dans l'immédiateté d'une première réception, de routines acquises principalement dans les fréquentations de programmes similaires. Ici aussi, la promesse du genre oriente l'usage. Ce qui n'est pas encore connu est ramené à une

\footnotetext{
J.-P. MEUNIER, op. cit., p. 74.

2 "Le topic est une hypothèse dépendant de l'initiative du lecteur" (U. Eco, op. cit., p. 116).
} 
classe de messages déjà identifiés et prédétermine les attitudes spontanées.

La rupture formelle et thématique de deux entités : le générique et le documentaire narratif sur l'aigle, ne pose pas problème aux enfants, habitués aux séries télévisuelles, où les génériques emblématiques d'une succession d'épisodes constituent des microrécits quasi autonomes. De même, la succession des scènes de vie : la chasse, le nourrissement des petits, leur envol, est rapidement agencée par référence aux documentaires animaliers.

La complexité de certains récits ne donne pas lieu à une structuration suivant des schémas actanciels ou narratifs, malgré un usage parfois abusif dans les classes, mais plutôt en microrécits juxtaposés ${ }^{1}$. Les plus importantes difficultés des enfants à organiser en grandes unités sont observées lors de confrontation à des tranches de flux télévisuels captées dans les émissions jeunesse comme celles de TF1. L'imbrication entre les programmes, les bandes annonces, les publicités, les séquences de présentations intermédiaires... est telle et à un tel rythme, qu'il faut provoquer plusieurs arrêts sur image, pour permettre aux élèves de repérer les transitions et délimiter les unités constitutives. Nous avons pu relever, à partir des commentaires des parents, que l'investissement pédagogique dans ce domaine produisait des répercussions notoires sur les pratiques télévisuelles familiales.

Les macroprocessus peuvent aussi concerner des unités plastiques (dominantes colorées, particularités graphiques de l'image) ou fonctionnelles (sources des images, lieux de tournage, atmosphères sonores). Cette capacité à saisir des effets de rupture et à constituer des ensembles par opposition, est très sensible à l'acte éducatif.

\section{e. Les processus d'élaboration}

Ils ont pour fonction de relier les informations tout au long de l'exposition au message en un réseau de cohérence global. A l'œuvre lors de la réception, ils se poursuivent bien au delà, les multiples opérations d'intellection qu'ils nécessitent sont tributaires des compétences en la matière du spectateur/lecteur. Le degré de sagacité de celui-ci peut prolonger l'action de ces processus vers l'infini des ouvertures potentielles de l'œuvre. On notera ici quelques unes de ces

1 Il est intéressant de noter que face à une impossibilité à raconter, un certain cinéma recourt aujourd'hui à une organisation fragmentée du scénario, semblable à cette attitude spontanée. 
opérations cognitives de niveau supérieur engagées à ce stade. Nous ne les décrirons pas avec précision car elles réfèrent au concept commun de compréhension, faisant fréquemment l'objet de présentation dans les ouvrages d'analyse de médias :

- l'établissement des conditions d'énonciations : finalités, partenaires, contexte... (Charaudeau, Soulages) ;

- la construction des mondes de référence et le régime de crédibilité à engager : authenticité, effet de réel, pertinence narrative, organisation artistique... (Jost) ;

- les prévisions et promenades inférentielles qui permettent d'ouvrir l'œuvre à la pluralité de sens (Eco) ;

- la recherche de cohérence par abduction créative : prolonger une fin ouverte, combler une ellipse énigmatique, synthétiser un documentaire...

- le repérage des structures axiologiques et idéologiques qui orientent le message ;

\section{f. Les processus de métacompréhension}

La plupart du temps ils œuvrent spontanément. Ils contribuent à la labilité permanente des premiers processus, de façon à ce que les concepts construits apparaissent comme recevables. Lors d'échecs répétés, les stratégies se conscientisent. Dans le générique alors que le lion est associé à une couronne, l'ours par fondu enchaîné provient de la forme stéréotypée d'une usine. La recherche de la métaphore sousjacente a donné lieu à diverses hypothèses, toutes jugées irrecevables par le groupe ${ }^{1}$. Les arguments déployés pour décider de cette irrecevabilité révèlent les stratégies individuelles de validation : sans appui sur les signifiants iconiques, sans cohérence avec le reste du générique, sans lien logique avec la vie de l'ours, sans pertinence avec l'intention des créateurs... Lors des séquences de sémiogenèse, la dynamique du groupe permet de partager les diverses compréhensions établies, mais aussi les stratégies qui ont permis de les établir ou de les vérifier. Ces temps de mutualisation sont déterminants dans les acquisitions procédurales individuelles. Ils favorisent par imitation, appropriation et assimilation les savoir-faire des pairs.

1 Cette construction renvoie à l'épisode correspondant sur l'ours, où cette usine produit une chaleur importante, à l'identique de l'animal qui survit dans des conditions glaciales. En l'absence de ce vécu, les enfants étaient contraints à des inférences très ouvertes. 
Ces processus permettent de valider ce qui est compris et de repérer les incompréhensions, mais aussi d'imaginer des ressources pour y remédier. Il est rapidement admis dans le réseau, qu'un support audiovisuel ou multimédia, quelle que soit sa valeur, ne peut représenter une entité absolue. Son contenu doit être mis en confrontation avec celui d'autres supports, la connaissance des ressources pertinentes en la matière, participe de cette compétence métacompréhensive'.

Ces processus interviennent aussi de façon décisive sur les potentialités à communiquer les compréhensions élaborées, en permettant leur formalisation langagière. L'intérêt du groupe est de socialiser ces connaissances individuelles, mais aussi de susciter une justification de leur organisation, voire une argumentation pour les faire valoir. Cette capacité à formaliser constitue la dernière étape indispensable, pour que la mise en œuvre d'un ensemble de stratégies de compréhension d'un message audiovisuel, conduise à la construction de réels savoirs.

\section{Conclusion}

L'ambition de ce modèle est son transfert dans le cadre de pratiques d'éducation aux médias. L'observation des productions de sens effectuées par des élèves de 8/11 ans dans le réseau, révèle son efficacité pragmatique. Loin d'être exhaustif, il permet cependant d'intégrer la plupart des attitudes et réactions des enfants offrant des participations langagières empiriquement mesurables sur le plan procédural. Il reste cependant à établir des protocoles d'observation et d'analyse, pour relier ces processus procéduraux avec les diverses structures à l'œuvre dans une démarche compréhensive, plus particulièrement sensori-motrices. Les modifications induites par une activité corporelle liée à l'approche du message audiovisuel : mime, chorégraphie, mise en scène thêâtrale, fiction adaptée en vidéo... renouvelle les interactions entre l'appréhension perceptive des messages, la façon d'en parler lors des productions langagières d'accompagnement (préparatifs, projets, évaluation des réalisations...) et la distanciation critique. L'intervention du corps transforme la pensée produite

1 La remise en cause de propositions du message, évaluées comme potentiellement fausses, engage aussi des stratégies de vérification et d'invalidation par raisonnement et confrontation documentaire, d'un grand intérêt sur le plan de la métacompréhension. 
jusque-là. L'incorporation de cette pensée modifie visiblement les paramètres des opérations mentales, de l'assimilation des données, et certainement des représentations internes qui en résultent. Autant de domaines qu'il reste à analyser et à opérationnaliser dans notre recherche-action, l'immensité du champ et la faiblesse de nos moyens nous renvoyant nécessairement à une attitude modeste quant aux objectifs.

L'autre orientation consiste à adapter le modèle aux variantes des hypermédias, à questionner plus finement leurs spécificités, notamment en reprenant les intuitions de G. Deleuze : "et l'écran lui-même, même s'il garde une position verticale par convention, ne semble plus renvoyer à la posture humaine, comme une fenêtre ou un tableau, mais constitue plutôt une table d'information, surface opaque sur laquelle s'inscrivent des «données», l'information remplaçant la Nature, et le cerveau-ville, le troisième œil, remplaçant les yeux de la Nature"'. La mutation de l'écran en tableau de bord, de l'image visible en image opérative, nous conduit nécessairement à reconsidérer certains processus (notamment sur le plan de l'élaboration et de la métacompréhension), même si de nombreuses vérifications à ce jour valident des pans entiers du modèle.

1 G. Deleuze, L'image-temps, Paris, Éd. de Minuit, 1985, p. 347. 


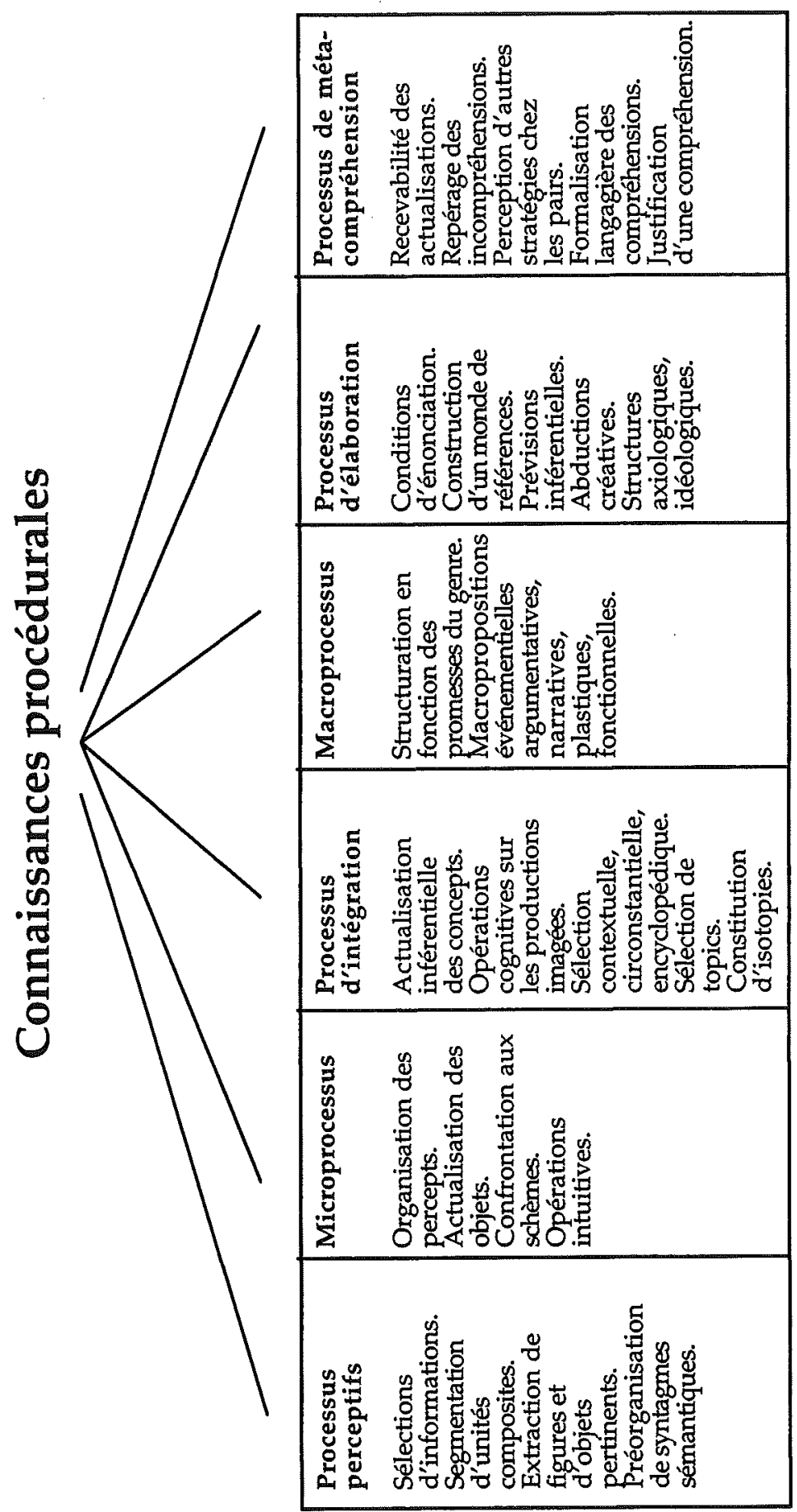

